\title{
Oportunidades para el cambio en la formación inicial docente desde las pedagogías feministas: educando en sexualidad e igualdad para docentes en formación
}

\author{
Irene Martínez Martín ${ }^{1 *}$, Virtudes Téllez Delgado² María Teresa Bejarano Franco ${ }^{3}$ \\ ${ }^{1}$ Universidad Complutense de Madrid, ${ }^{2}$ Universidad Autónoma de Madrid, ${ }^{3}$ Universidad de Castilla La Mancha
}

\author{
PALABRAS CLAVE \\ Educación \\ Sexualidad \\ Pedagogía feminista \\ Formación profesorado \\ Igualdad
}

\author{
KEYWORDS \\ Education \\ Sexuality \\ Feminist pedagogy \\ Teacher training \\ Equality
}

\begin{abstract}
RESUMEN
Las pedagogías feministas posicionadas en paradigmas críticos y postcríticos de la educación buscan transformar las estructuras que generan desigualdad y exclusión en los procesos de enseñanza y aprendizaje. Este artículo pone el foco en la educación en sexualidad e igualdad como objeto de estudio y propuesta de transformación dentro de las pedagogías feministas. Queda enmarcado en una investigación llevada a cabo en la UCLM y en la que se ha aplicado la triangulación de tres técnicas: análisis documental de planes de estudio y legislación, cuestionarios a estudiantes de últimos cursos de los grados de educación, entrevistas y técnica Delphi a profesorado experto en las temáticas señaladas. Destacan resultados encaminados a evidenciar la falta de una educación en sexualidad de calidad en la formación docente, la reproducción de roles, estereotipos y mitos en relación a la sexualidad y los frenos y retos en la inclusión de la sexualidad en las dimensiones científicas y académicas universitarias. Como principales propuestas se busca dar respuesta desde las pedagogías feministas a la construcción de un modelo de educación en sexualidad e igualdad para la formación docente.
\end{abstract}

Opportunities for change in initial teacher training from feminist pedagogies: training in sexuality and equality for teachers in training ABSTRACT

Feminist pedagogies positioned in critical and post-critical paradigms of education seek to transform the structures that generate inequality and exclusion in the teaching and learning processes. This paper focuses on education in sexuality and equality and to propose feminist pedagogies. It is framed in a research carried out at the UCLM and in which the triangulation of three techniques has been applied: documentary analysis of study plans and legislation, questionnaires for students in the last years of education degrees and interviews with expert teachers in the main topics. Results highlighted the lack of quality sexuality education in teacher training, the reproduction of roles, stereotypes, and myths in relation to sexuality and the obstacles and challenges in the inclusion of sexuality in university scientific and academic dimensions. The main proposals seek to respond from feminist pedagogies to the construction of a model of education in sexuality and equality for teacher training.

Universidad Complutense de Madrid

Autora de correspondencia: * Irene Martínez Martín. imarti02@ucm.es

Recibido: 07/05/2021 - Aceptado: 09/07/2021

Revista de Formación del Profesorado e Investigación Educativa

Facultad de Formación del Profesorado y Educación

Universidad de Oviedo

Enero - Diciembre 2021

ISSN: $2340-4728$

(c) (1) () $\odot$ Esta obra está bajo una licencia internacional Creative Commons

Atribución-NoComercial-SinDerivadas 4.0 . 


\section{Introducción y marco teórico}

¿Qué tipo de educación en sexualidad e igualdad se da en las diferentes etapas educativas? ¿Desde la educación, reproducimos o transformamos las prácticas de desigualdades y violencias machistas, derivadas de la falta de una educación en sexualidad e igualdad de calidad? ¿Qué necesidades tiene el profesorado para desarrollar una educación en sexualidad e igualdad desde perspectivas feministas? ¿Es posible despatriarcalizar el currículum escolar y construir un currículum desde las pedagogías feministas?

Estas, y otras muchas cuestiones, abren las reflexiones que se aportan en este artículo. Como finalidad, este escrito busca cuestionar los modelos educativos en torno a las sexualidades y la igualdad que reproducen estereotipos, roles y violencias machistas en la educación. Según Segato (2018) el patriarcado es la primera escuela de poder, de jerarquía, de homogeneidad y expropiación de la diversidad, de lo colectivo y lo individual. Tiene, además, un fiel aliado en los sistemas educativos hegemónicos.

En concreto, este artículo pone en el centro del análisis la educación en sexualidad e igualdad recibida a lo largo de todas las etapas educativas. En este sentido, se destacan varias dimensiones de estudio: 1) aprendizajes formales y no formales que contribuyen a construir la sexualidad individual y colectiva; 2) los mitos y estereotipos machistas que limitan la sexualidad a la genitalidad heterosexual; y 3) la imposición de la moral y lo privado como creencia dominante en la educación sexual. El conjunto de estas dimensiones, desde un análisis crítico de las pedagogías feministas, ayuda a visibilizar las dificultades encontradas en el desarrollo pleno de una educación sexual integral y que vaya más allá de un conjunto de informaciones sobre el sexo y las relaciones sexuales (heterosexuales, genitales, enfocadas al embarazado, sin valorar el autoconocimiento ni el respeto de los límites, propios y ajenos, como tampoco entiende ni asume las diversidades).

El cuestionamiento de estas dimensiones es el punto de partida de la investigación aquí presentada y tienen como finalidad la construcción de una educación feminista, considerando la formación inicial del profesorado como elemento central.

\section{Construcción de la educación en sexualidad desde los discursos oficiales}

El marco de partida supera los discursos reduccionistas de la sexualidad centrados en la dimensión biológica como único eje visible. Estos posicionamientos hacen que la educación en sexualidad quede limitada a contenidos de asignaturas de ciencias naturales y como aspectos meramente físicos. Se imponen visiones hegemónicas y estereotipadas en las que predominan los posicionamientos desideologizados. Aprendizajes que dejan fuera las identidades diversas, el autoconocimiento feminista, los cuidados y los buenos tratos, así como afectos, expresiones corporales y emocionales. Esta visión, fuertemente arraigada en los currículums educativos, invisibiliza las identidades sexuales diversas y refuerza mitos y estereotipos sexistas en relación a la vivencia de la propia sexualidad (y la sexualidad del otro/a), y deja la responsabilidad educativa en manos de la moral y los ámbitos no formales y privados (religión, familia, redes sociales, pornografía o grupo de iguales); teniendo como resultado la invisibilización de las sexualidades en los ámbitos educativos formales.

Así, Bejarano y García (2016) afirman que es muy difícil encontrar experiencias verdaderas de educación afectivo-sexual pese a las recomendaciones de la UNESCO. Insisten en señalar que los conocimientos sobre sexualidad, en España, se han impartido desde la imposición biologicista separándose de los contenidos vinculados con la afectividad, las identidades y las emociones.

Entender la educación en sexualidad (Bejarano y Marí, 2019) como un aspecto integral, diverso y político, supone romper las barreras de lo íntimo y privado asumiendo que no solo se lleva a cabo en las instituciones educativas, sino que toda sociedad educa sexualmente a sus miembros como parte de los procesos de socialización, mediante la cultura, la familia, los medios de comunicación, las religiones, las producciones audiovisuales, las redes sociales, entre otras. Por tanto, la sexualidad y su educación tienen poco de íntimo y privado. Al contrario, como diría Freire (1967), la educación no es neutra, es una práctica política de acción, libertad y transformación sobre el mundo.

La educación en sexualidad desde esta perspectiva multidimensional y social está en consonancia la Organización Mundial de la Salud [OMS] $(2018$, p.3):

Un aspecto central del ser humano que está presente a lo largo de su vida. Abarca el sexo, las identidades y los roles de género, la orientación sexual, el erotismo, el placer, la intimidad y la reproducción. Se siente y se expresa a través de pensamientos, fantasías, deseos, creencias, actitudes, valores, comportamientos, prácticas, roles y relaciones. Si bien la sexualidad puede incluir todas estas dimensiones, no todas ellas se experimentan o expresan siempre. La sexualidad está influida por la interacción de factores biológicos, psicológicos, sociales, económicos, políticos, culturales, éticos, legales, históricos, creencias religiosas y espirituales.

También, en esta misma línea, cabe destacar los aportes de la UNESCO (2014, p. 37):

La sexualidad es parte integral de la vida de las personas (en sus diferentes etapas o edades, y en toda su diversidad), y contribuye al desarrollo de su identidad y, por lo tanto, a su desarrollo social. Se trata de capacidades más que de contenidos, las que deben desarrollarse de forma interdisciplinaria y de manera progresiva para que las personas actúen de manera responsable respecto a ellas mismas y con los demás.

Todo ello va conformando unas narrativas oficiales y formales en las que la educación en sexualidad e igualdad rompe con los viejos tabúes y estereotipos patriarcales, ya mencionados, y avanza hacia unos modelos integrales. Estos principios globales tienen su reflejo en las legislaciones y políticas educativas en Europa y América Latina, especialmente llamativos son algunos programas del territorio español como Skolae, la propia LOMLOE o el programa ESI en Argentina.

\section{Educación en sexualidad desde narraciones feministas contrahegemónicas}

Investigaciones como las de Eisenstein (2007), Platero (2014), Sánchez (2020), Enguix y González (2018) cruzan los elementos educativos formales con las narraciones feministas y la construcción y vivencias de la sexualidad. En todas ellas se desvela la necesidad de introducir las competencias en igualdad y sexualidad en los espacios de aprendizaje para identificar y erradicar las consignas patriarcales, así como incluir el principio de igualdad en todo el proceso. Se considera la educación en sexualidad e igualdad desde el enfoque de derechos humanos ya que atiende al desarrollo de las necesidades básicas, el respeto a las propias identidades y a la construcción de ciudadanías basadas en la convivencia y los buenos tratos. En este sentido se tiene en cuenta que la dimensión sexual incluye: el autoconocimiento, la salud, la intimidad, los afectos, los cuerpos, el deseo, la comunicación, la seguridad, la convivencia, la expresión de límites y aceptación de estos, el amor y el buen trato, entre otras. (Fallas et al., 2012).

Sumando a este marco formal donde la educación en sexualidad e igualdad rompe los límites de lo puramente biológico encontramos los aportes que desde los feminismos críticos se hacen. Platero (2014) afirma la necesidad de incluir un enfoque interseccional en los aspectos relacionados con las identidades sexuales y de género. Además, apuesta por la importancia de incluir estos 
aportes en la formación de profesorado como aliado para actuar frente a las violencias, las imposiciones patriarcales y la reproducción de roles y estereotipos binarios.

Señala Butler (2004) que una noción universal de género y sexualidad puede ser una forma más de cultura imperialista y colonizadora, por ello necesitamos una comprensión plural e interseccional de las culturas, los géneros, las sexualidades y la igualdad.

Desde este marco de narrativas feministas contrahegemónicas se refuerza la concepción de la sexualidad desde la multidimensionalidad, como un aspecto dinámico y en continua construcción, y que es político. Además, se ajusta a los principios de diversidad que tratan de romper con los binarismos impuestos donde lo masculino se asocia a unos estereotipos marcados y entendidos como normativos (al igual que ocurre con los femeninos). Este binarismo es jerárquico, desigual y generador de violencias, al imponer lo masculino-hegemónico como lo normativo y superior, dejando en planos de inferioridad y opresión todas las disidencias y desigualdades.

Bakare-Yusuf (2013) señala lo sexual masculino-hegemónico como una herramienta política para mantener el estatus de poder que, además, es aprendida e impuesta. Todo ello provoca que la sexualidad no heteronormativa se aprenda y se viva bajo el reduccionismo, las violencias y la invisibilización de deseos y cuerpos.

Incluir los aspectos aquí descritos, la sexualidad y la igualdad como derecho y desde una perspectiva interseccional, es un reto que abordar desde las instituciones universitarias para, por un lado, visibilizar las carencias y desigualdades y, por otro lado para avanzar en las reformas didácticas y discursivas en favor del principio de igualdad. Establecer paralelismos y puntos de encuentro entre las narrativas institucionales y formales con las narrativas feministas y activistas es un compromiso de las pedagogías feministas (Martínez y Bejarano, 2021). Estas narrativas aportan como retos para transformar la educación en sexualidad los siguientes: avanzar en las modificaciones de los planes de estudio en favor de la promoción de la igualdad y la educación en sexualidad; incluir estas temáticas como elementos científicos; y avanzar hacia el compromiso de educar en ciudadanías feministas, críticas, en equidad y con justicia social.

Como consecuencia las pedagogías feministas apuestan por formar al profesorado de los Grados de Educación en estas cuestiones, ya que tendrán responsabilidad futura en la transformación de las desigualdades y violencias, introduciendo en el sistema educativo saberes sobre igualdad y educación en sexualidad (Barber y Mourshed, 2008; Sánchez, et al., 2014) desde el respeto a las diversidades.

\section{Objetivos}

La investigación aquí presentada cumple con tres grandes objetivos derivados del marco teórico presentado y acorde a los retos planteados por las pedagogías feministas:

1) Analizar el conocimiento de los y las estudiantes de cuarto curso de los grados de Educación en relación con la igualdad y sexualidad

2) Visibilizar las narrativas del profesorado respecto al rol de la Universidad en la promoción de la igualdad y la sexualidad (docencia e investigación);

3) Conocer si los planes de estudio de Educación (España) recogen competencias curriculares sobre igualdad y sexualidad.

Se suma, además, un cuarto objetivo que pretende triangular la información obtenida del estudiantado, el profesorado y los planes de estudio. Todo ello para establecer un diagnóstico inicial de la situación de la educación en sexualidad e igualdad en las universidades españolas desde una perspectiva feminista.

\section{Método de investigación}

Se presentan aquí los análisis derivados de la triangulación de datos obtenidos de tres instrumentos: análisis de planes de estudio, cuestionarios a estudiantes y entrevistas a profesorado.

\section{Análisis de planes de estudio y legislación}

El análisis de los planes de estudio se realizó (Martínez, et al., 2017) siguiendo una hoja de registro en base a dos niveles de análisis: 1) existencia de competencias en igualdad y sexualidad; y 2) existencia de la perspectiva de género de manera directa en tres niveles: asignaturas específicas de género; inclusión de contenidos específicos de género; o contenidos transversales de género.

Este instrumento de registro tuvo como palabras clave relacionadas con la temática: sexualidad, género, igualdad, desigualdad, identidad, violencia y machismo. Todo ello fue aplicado tanto a las competencias generales y transversales de los Grados de Educación, como a las memorias de los planes de estudio y guías docentes. También se realizó este análisis a la legislación educativa europea, nacional y regional.

\section{Cuestionarios a estudiantes de cuarto curso de los grados de educación}

Para la aplicación de los cuestionarios a estudiantes del cuarto curso de los Grados de Educación se contó con un instrumento construido a partir de un cuestionario inicial aplicado en una investigación de Veiga et al. (2006) y que fue ampliado y validado por el grupo GIES (Grupo de Investigación en Educación y Sociedad) de la Universidad de Castilla La Mancha (UCLM).

Dicho instrumento cuenta con nueve dimensiones en las que se recoge información sobre: $1^{\circ}$. Datos personales. $2^{a}$. Formación institucional. $3^{\text {a }}$. Conocimiento científico básico. $4^{\mathrm{a}}$. Comportamientos sexuales. $5^{\mathrm{a}}$. Motivación para las relaciones sexuales. $6^{\mathrm{a}}$. VIH SIDA. 7a. Identidad y orientación sexual. $8^{a}$ Violencia sexual. 9a. Creencias sobre sexualidad. Cada dimensión muestra un banco de preguntas de respuesta múltiple, en total 57.

La muestra se compuso por 371 personas, todas ellas estudiantes de cuarto curso de las seis Facultades de Educación y Educación Social de la UCLM. Se tomó como referencia un nivel de confianza del 95,5\%, asumiendo un error muestral del 5\% y utilizando como estimador de varianza poblacional $p=q=0,50$. Esta muestra se caracteriza por su feminización y juventud, tres de las cuartas partes de las respuestas $(78 \%)$ pertenecen a mujeres estudiantes, y en nueve de cada diez ocasiones $(88,4 \%)$ tienen 25 años o menos.

El análisis del cuestionario se realiza mediante un estudio estadístico de tipo descriptivo por frecuencias básicas y, después, por un análisis de correlaciones. Así, se aporta información acerca del nivel de conocimiento en torno a la sexualidad y la igualdad y las conductas vinculadas a las identidades sexuales.

\section{Entrevistas en profundidad a profesorado experto de la UCLM}

Las entrevistas semiestructuradas y técnica Delphi fueron aplicadas a una muestra de 32 profesionales universitarios, expertas en temáticas de género y sexualidad de las titulaciones de Educación de la UCLM. La selección fue no probabilística, siendo intencional y utilizándose como criterios para garantizar la representatividad de la muestra: la experiencia en la temática de la investigación y la distribución equitativa entre los distintos campus universitarios de la UCLM.

El guion de la entrevista cuenta con 16 preguntas semiestructuradas y un cuestionario Delphi con nueve apartados de preguntas abiertas. Este cuestionario Delphi busca confirmar aquellos datos y discusiones analizadas en las entrevistas (realizadas previamente). 
Tanto las entrevistas como los cuestionarios Delphi se estructuran en torno a tres dimensiones o categorías de análisis: 1) existencia de la perspectiva de igualdad y sexualidad en un nivel institucional-universitario; 2) nivel de conocimientos del profesorado universitario en relación con las temáticas de sexualidad e igualdad y 3) aplicación docente e investigadora real de los contenidos relacionados con la sexualidad y la igualdad. El análisis de las entrevistas y Delphi se hace de manera conjunta en base a una tabla de multicategorías de análisis y apoyándose en el programa Atlas. Ti.

\section{Triangulación de resultados y discusión}

Se presentan los principales resultados obtenidos a partir del proceso de triangulación de las técnicas utilizadas. Se va a realizar una descripción reflexiva del cruce de todas las dimensiones estudiadas, atendiendo a las principales categorías de análisis, divididas en dos grandes bloques: frenos y oportunidades para desarrollar la educación en sexualidad e igualdad.

Bajo conocimiento y falta de representación de conocimientos en sexualidad e igualdad

En primer lugar, es destacable el desconocimiento desde un enfoque formal, científico, riguroso y feminista de los contenidos básicos de la educación en sexualidad e igualdad. En este sentido, en los planes de estudio de los Grados de Educación no aparecen contenidos específicos, asignaturas concretas (salvo alguna optativa puntual) ni competencias directas. Es decir, hay una falta de representación de los contenidos clave de educación en sexualidad e igualdad. Tampoco, en las guías docentes de los Grados de la UCLM se recogen contenidos relacionados con ambas temáticas.

Esto contrasta con los avances a nivel legislativo que sí contemplan estas temáticas como elementos centrales para su abordaje educativo. También, da lugar a una diversidad de experiencias, más o menos globales, a lo largo de todo el territorio español a la hora de implementar programas de educación en sexualidad e igualdad.

Esta inexistencia de las temáticas de género y sexualidad en los planes de estudio se evidencia en la falta de conocimiento básico por parte del alumnado de $4^{\circ}$ curso de los Grados de Educación. Así, se refleja en los resultados de los cuestionarios dimensiones 1 y 2 en los que se dan respuestas confusas a las cuestiones relacionadas con: los ciclos menstruales, las posibilidades de embarazo y la transmisión de enfermedades, el deseo o las identidades sexuales. Aparece con mayor claridad la perspectiva multidimensional de la sexualidad al tener claro, el estudiantado, la dimensión afectiva, emocional y de autocuidados.

También, en este sentido, el profesorado entrevistado afirma que la educación en sexualidad e igualdad es débil y depende mucho del interés particular de los propios docentes. Al no haber un plan de estudios ni interés real de la institución universitaria en estas temáticas, quedan excluidas de los programas oficiales y solamente se incorporan si el profesorado se muestra con interés o es experto en la materia.

El proceso para introducir la perspectiva de género (igualdad y sexualidad) en estos planes de estudio obedece fundamentalmente a introducir asignaturas optativas, que están incluidas, por sensibilización e implicación de docentes en concreto que tienen interés en estas temáticas y esta materia esté presente en la formación (P.20 V3 INF).

Este desconocimiento manifiesto, tanto por el profesorado como por el alumnado y planes de estudio, se solventa en la mayoría de las veces con las redes informales de educación (grupo de iguales, redes sociales, pornografía, religión, familia...). No todas estas redes informales son negativas, pero sí redundan en el hecho de la falta de un discurso crítico en materia de género y en el debate de contraponer la educación en sexualidad como un aspecto moral y privado que no debe ser atendido desde las instituciones educativas.

Falta de una cultura de género e implementación "cosmética" de la perspectiva de género en la vida académica

Es un análisis común (planes de estudio, entrevistas y cuestionarios) el uso generalizado de lenguaje sexista, por ejemplo, en las guías docentes usando generalizaciones tales como "los niños", "los hombres"; haciendo uso del lenguaje masculino-excluyente; en la impartición de la docencia; o en los materiales utilizados (falta de representación femenina en lecturas, sesgos en la representación de contenidos, etc.).

... Se tienen que formar para poder ser referencia para sus propios alumnos y alumnas en un futuro y ser alguien a quien ese alumnado se pueda dirigir en casos también de necesidad o de acoso, o de violencias, o simplemente de duda, o de bueno, pues que van a ser figuras de referencia (P.13 V1 INF).

En este sentido se detecta una contradicción entre los mandatos de leyes específicas en materia de igualdad y violencia de género, a nivel estatal, y los planes de estudios que forman a maestros y maestras, educadores y educadoras sociales, ya que no reflejan los contenidos específicos de igualdad y sexualidad, o quedan reducidos a menciones generales, transversales o relegadas a los intereses particulares.

Destaca, sobre todo en las entrevistas, que estamos ante un tema de moda. Suscita interés entre el estudiantado, se dan cada vez más investigaciones y publicaciones, jornadas y eventos que tienen como motor el género. Aunque se refieren también a la falta de perspectiva crítica y científica y el riesgo que supone despolitizar un tema como el feminismo, o la sexualidad, por el hecho de ser una moda. Este peligro supondría no materializar esta gran producción actual en cambios estructurales profundos en el ámbito académico y legislativo.

En la academia nos queda mucho y no sé hasta qué punto no estamos precisamente en un momento de...de reacción, que está sucediendo fuera de la academia y que nos está llegando también a la académica (P.6 V2 INF).

Peso de los sesgos, prejuicios, falsas creencias y estereotipos que rodean a la igualdad y la diversidad sexual

Los hallazgos obtenidos en los instrumentos de investigación advierten que la educación en igualdad y sexualidad son temas que no se trabajan significativamente. Es por ello que se hacen presentes estereotipos y mitos, principalmente referidos a las identidades diversas.

En este sentido, la calidad del aprendizaje y su valoración en materia de igualdad y sexualidad es cuestionable, tal y como reflejan los resultados de los cuestionarios. Aunque el contacto con la educación en igualdad de oportunidades y la diversidad sexual gana cierto espacio, las aproximaciones a los contenidos adquieren todavía un carácter muy puntual sin atender a un itinerario explícito. Es decir, aparecen dudas en relación con conceptos como la transexualidad, la identidad sexual, las expresiones de género o sus respuestas educativas.

...lo que parece es que sólo existe un modelo de sexualidad que es el hegemónico, hay que tratar esos temas para que los conozcan sin los prejuicios ... que tengan una visión distinta para que luego puedan aceptar a la gente que tenga en las aulas desde otras opciones y otras circunstancias ... el respeto hacia esas actitudes son básicas para luego integrar y anclar esa 
actitud multidimensional ya creada con conocimientos de la realidad pero desde una mirada diversa pero con tratamiento específico para que se sepa que es una práctica social-cultural como otra más y que cada grupo social puede desarrollar de distintas maneras (P.36 V1-V2, V4 INF).

A su vez, en las entrevistas destaca por un lado una preocupación por la rigidez curricular, que impediría la inclusión de este tipo de contenidos. Por otro lado, también se menciona la dejadez y la transversalidad mal entendida, como excusa, para no tratar estos temas de manera directa o tratarlos insuficientemente y sin el rigor científico y actualidad que requieren.

Falta de apoyo explícito a la temática y a los colectivos feministas dentro de la investigación universitaria

Los avances académicos y formativos son débiles, aspecto que contrasta con el protagonismo reivindicativo de los movimientos sociales feministas.

Si yo estoy haciendo Género tengo que decir "estoy trabajando Género" y estoy interviniendo con una forma de ver esto y lo tengo que verbalizar y tomar partido delante de los alumnos (P.15 V3 INF).

Además, se siguen reproduciendo estereotipos de género, mitos sobre la sexualidad e invisibilizando las diversidades, todo ello teniendo como consecuencia no llevar a la práctica una educación feminista transformadora en los ámbitos universitarios.

He sido autodidacta, mi formación, por así decirlo académica, institucional, no existe... (P.9 V4 INF).

$Y$ las creencias sobre la sexualidad, yo estoy convencida que la mayoría, incluida las nuestras, podemos ser partícipes de creencias erróneas respecto a lo que es educación en sexualidad (P.25 V1 INF).

En las entrevistas al profesorado se aprecia un alto interés por la temática, pero una preocupación por su falta de apoyo y marco científico. La educación en igualdad y sexualidad es considerada una materia indispensable para la formación de los y las futuras docentes. Se interpela a la Universidad como agente de cambio social a potenciar y utilizar la institución como impulsora de innovación social, también en temas de género y sexualidad. Esto pasa por corregir los actuales déficits de la oferta, muy estacional y modular, insuficiente y que no brinda el acervo teórico ni las herramientas científicas para enfrentar y resolver situaciones cotidianas que surgen en los entornos educativos (Bejarano y Martínez, 2020).

\section{Conclusiones: Oportunidades para el cambio desde las peda- gogías feministas}

Cabe destacar que estos resultados son coincidentes con otros estudios e investigaciones (Ballarín, 2017; Bejarano y Marí, 2019; Bejarano y Martínez, 2020; Sánchez, 2018; Subirats, 2017; Bejarano, Martínez y Téllez, 2021) donde se evidencia que la educación en igualdad y sexualidad es débil o inexistente en la formación del profesorado.

La investigación, revela una confusión generalizada del futuro profesorado respecto a las cuestiones que constituyen la educación en sexualidad e igualdad; ello puede derivar en el desarrollo de prácticas profesionales lineales, binarias y poco inclusivas ante la diversidad y orientación sexual. La aceptación social de la realidad sexual en los términos emergentes en que ha sido discutida en este artículo, es requisito indispensable para desarrollar una educación feminista como respuesta ante los indicios sobre los niveles de violencia ejercida a quienes no se ubican en el marco heteronormativo. Así lo desvelan diversos informes publicados recientemente, que exponen el carácter sistémico de la violencia por orientación sexual o identidad de género en los centros de enseñanza españoles (García et al., 2011; Benítez, 2016).

También, teniendo en cuenta las entrevistas del profesorado como respuesta a las carencias del estudiantado (reflejadas en los cuestionarios y planes de estudio) cabe destacar una serie de oportunidades que potencien los cambios necesarios. Referida a la práctica docente universitaria en igualdad y diversidad afectivo-sexual destaca la mayor sensibilización y predisposición hacia estas temáticas entre los distintos sectores sociales. Todo ello queda avalado en otras investigaciones similares (Bejarano y Marí, 2019; Castaño, 2016; Martínez y Ramírez, 2017; Pastor y Acosta, 2016; Teixeira y Marques, 2016; Sánchez et al. 2016; Sánchez, 2019).

En este contexto, se considera la potencialidad para mejorar el currículo formativo al haber un creciente compromiso por la formación permanente y tener la sexualidad y la igualdad en agenda. Se considera que los planes de estudio, así como la formación del profesorado, deben ser revisados desde los postulados feministas, interseccionales, decoloniales y críticos de la pedagogía para la inclusión de las diversidades (Bejarano y Marí, 2019; Martínez, 2018), sumando, a su vez, metodologías reflexivas, críticas y colaborativas en la formación docente que ayuden a repensar y revolucionar la cultura pedagógica, sus teorías y prácticas.

Construir una formación del profesorado feminista (Bejarano et al., 2019) es apostar por marcos normativos y reguladores favorables (en muchos casos ya existentes, pero con dificultades para su puesta en práctica); es alentar una verdadera educación crítica y transformadora en todos los espacios y estructuras educativas, incluyendo mayor actividad investigadora y de calidad (consolidando Tesis, Trabajos Fin de Máster, Trabajos Fin de Grado o proyectos de investigación competitivos, entre otros).

\section{Propuesta: decálogo para una formación del profesorado en se- xualidad e igualdad desde las pedagogías feministas}

Corroboramos que no se prepara convenientemente a los profesionales españoles de la docencia para desarrollar una educación en sexualidad e igualdad de calidad, en definitiva, capaz de reconocer la diversidad afectivo-sexual, prevenir la violencia y los riesgos que esta conlleva, o ser capaces trasladar pautas de vida democráticas y respetuosas con el principio de igualdad.

Ramos et. al (2020) y Martínez (2016) proponen despatriarcalizar la educación a partir del siguiente decálogo:

1) Cuestionar los contextos que habitamos. Es decir, qué modelos, contenidos, imágenes, narrativas, lugares simbólicos, espacios, lenguajes... son aquellos que nos construyen como ciudadanos y ciudadanas. Ante estas evidencias, las pedagogías feministas tienen una exigencia básica: deconstruir los aprendizajes basados en la socialización diferenciada, así como sus mecanismos de reproducción y de sanción. Se posicionan como lucha política por la igualdad y reclaman a la educación ese componente político de transgresión y transformación de las estructuras de desigualdad.

2) Descolonizar la educación desde posicionamientos antirracistas e interculturales. Aprender a ser, a convivir, a hacer y conocer rompiendo con la sobrerrepresentación de un único discurso (blanco, masculino, clase media-alta, heteropatriaracal, etc.) y abrir escenarios para poner en diálogo formas alternativas y diversas de estar en el mundo.

3) Pedagogías situadas, contextualizadas y territorializadas. No hablamos de una única receta cerrada, ya que la educación no es neutra y va a depender de contextos, tiempos y espacios determinados. Una educación comunitaria que nos interpele como parte de un territorio desde la responsabilidad y el bien común. Una educación que enseñe a transgredir, que no suponga únicamente el acceso 
al poder, sino también el uso transformador de los poderes colectivos y liderazgos compartidos. Esto nos lleva a una educación en la participación plena y consciente, y a la acción organizada desde lo colectivo.

4) Pedagogía como resistencia ante las violencias y como contra-pedagogía a los mensajes de odio y de poder patriarcal. Una educación anti-patriarcal que cuestione los mandatos de la masculinidad hegemónica y todo lo que de ello se deriva (colonialismo de un único tipo de saber, valores asociados a la competitividad y la agresividad, desensibilización, etc.).

5) Educación en la memoria histórica, en la recuperación de los saberes ancestrales y en la visibilización de genealogías feministas e interseccionales. Supone conocer y conectar de dónde venimos y poner en valor conocimientos y experiencias diversas como acto de justicia, memoria y reparación.

6) Educación en masculinidades no hegemónicas que visibilicen cómo el machismo también victimiza, silencia y oprime las masculinidades y donde se nombre la violencia en todas sus dimensiones, intragénero e intergénero.

7) Pedagogía del cuidado. Cuidar como acto de transgresión ante los valores neoliberales que imponen el individualismo, el egoísmo y el materialismo. Supone educar en lo colectivo, en los buenos tratos y en lo comunitario estableciendo vínculos recíprocos en libertad e igualdad y poniendo límites a la cosificación de la vida, a la degradación de los y las otras y, en definitiva, a las relaciones de violencia. También, el cuidado en los límites y normas, en las relaciones de autoridad y en los repartos de poder.

8) Educación interseccional como cambio de enfoque educativo. Supone empezar a entender la diversidad como lo normativo y no como lo extraño que se señala, se excluye y se silencia o violenta. Es decir, las diferencias como algo dinámico y donde las opresiones y privilegios contribuyen a generar nuestra mirada de lo "otro" y deben ser repensadas y deconstruidas.

9) Despatriarcalizar el curriculum educativo. Atendemos a los contenidos, lenguajes, metodologías, espacios y sistemas de evaluación. Todo ello desde una necesidad de deconstruir para volver a construir desde los feminismos, apostando por metodologías cooperativas, contenidos descolonizados y despatriarcalizados, lenguajes inclusivos, evaluaciones formativas no excluyentes y la organización de los espacios, tiempos, mobiliarios, patios... situando los cuidados en el centro y la pedagogía dialógica y participativa sin condicionantes de género.

10) Implicar los cuerpos, las emociones y afectos en la educación. Educación en sexualidad e igualdad que tenga en cuenta los saberes básicos, pero desde perspectivas de diversidad, incluir referentes de todas las identidades, repensar las imposiciones y sesgos de género, romper estereotipos y mitos, superar tabúes y empoderar desde la adquisición de herramientas y estrategias individuales y colectivas para la transformación.

Se trata, por lo tanto, de construir una educación feminista que tenga en cuenta incluir una pedagogía de las resistencias antes las violencias, una pedagogía de la escucha que sitúe los cuidados feministas en el centro, una pedagogía comunitaria y popular y una pedagogía dialógica, participativa, social y colectiva; debe ser el reto para asumir, también, desde las estructuras universitarias en docencia e investigación.

El tipo de historias que contemos sobre nuestras vidas sexuales son importantes. Contar historias positivas sobre mujeres que viven su sexualidad de manera libre, importa, porque ayuda a reconstruir, empoderar y recordar que la sexualidad de las mujeres (hombres y disidencias) no es un universo simplificado de miedos, errores, violencia y negaciones (Bakare-Yusuf, 2013, p. 37).

\section{Agradecimientos}

Este artículo muestra una síntesis de los resultados obtenidos en el proyecto de investigación llevado a cabo por el grupo GIES de la Universidad de Castilla la Mancha en los años 2016-2018, bajo el título: "La educación en sexualidad e igualdad en la formación inicial del profesorado y educadores/as sociales" financiado por el Instituto de la Mujer de Castilla la Mancha y la Universidad de Castilla la Mancha.

\section{Contribución individual de las autoras al manuscrito}

IMM ha contribuido en la organización y coordinación de este artículo, así como en los análisis previos de la investigación desarrollada y en la construcción del marco teórico y las conclusiones y propuestas en torno a las pedagogías feministas. MTB ha contribuido como investigadora principal de los proyectos de investigación que han dan como resultado esta producción, así como en los análisis de los instrumentos de recogida de datos y en la construcción del marco teórico. VTD como antropóloga ha contribuido en los análisis cualitativos de las entrevistas, así como en la redacción de resultados y conclusiones de este artículo.

El trabajo de redacción y discusión ha sido conjunto, así como el trabajo en equipo que se desprende de todas las fases previas de investigación y estudio que tiene como resultado un artículo como este.

\section{Referencias bibliográficas}

Ballarín, P. (2017). ¿Se enseña coeducación en la Universidad? Atlánticas. Revista Internacional de Estudios Feministas, 2(1), 7-31. https://doi.org/ 10.17979/arief.2017.2.1.18652017

Bakare-Yusuf, B. (2013). Thinking with pleasure. danger, sexuality and agency. En S. Jolly, A. Cornwall y K. Hawkins (ed.), Women, sexuality and the political power of pleasure (pp. 28-57). Zed Book.

Barber, M. y Mourshed, M. (2008). Cómo hicieron los sistemas educativos con mejor desempeño del mundo para alcanzar sus objetivos. Mckinsey y Company. Preal.

Benítez, E. (Coord.)(2016). LGBT-fobia en las aulas 2015. ¿Educamos en la diversidad afectivo sexual? Grupo de Educación de COGAM.

Bejarano M. T. y García, B. (2016). La educación afectivo-sexual en España. Análisis de las leyes educativas en el periodo 19902016. Opción, 32 (13), 756-789.

Bejarano, M.T y Marí, R. (2019). Educación en sexualidad e igualdad. Dykinson.

Bejarano, M.T; Martinez, I. y Blanco, M. (2019). Coeducar hoy. Reflexiones desde las pedagogías feministas para la despatriarcalización del curriculum. Revista Tendencias Pedagógicas, 34, 37-50. https://doi.org/10.15366/tp2019.34.004

Bejarano, M. T. y Martinez, I. (2020). Propuestas desde las pedagogías feministas para despatriarcalizar la educación. Revista Diversidade e Educaçao, 8 (especial), 1-9. https://doi.org/10.14295/ de.v8iEspeciam.11044

Bejarano, M.T.; Martínez, I. y Téllez, V. (2021). Narrativas del profesorado universitario en sexualidad e igualdad. Revista Athenea Digital, 21 (3), e3041-e3041.

Butler, J. (2004). Deshacer el género. Paidós.

Castaño, C. (2016). La nueva gestión pública y las políticas de igualdad de género en las universidades. Investigaciones feministas, 7(2), 225-245.

Eisenstein, Z. R. (2007). Sexual Decoys. Gender, race and war. Zed books.

Enguix, B. y González, A.M. (2018). Cuerpos, mujeres y narrativas: imaginando corporalidades y género. Revista Athenea digital, 18(2), 1-31. https://doi.org/10.5565/rev/athenea.1956 
Fallas, M.; Atarvia, C. y Gamboa, A. (2012). Educación sexual: Orientadores y orientadoras desde el modelo biográfico y profesional. Revista Electrónica Educare, 16 (Especial), 53-71.

García-Pérez R.; Rebollo Á.; Vega, L.; Barragán-Sánchez, R.; Buzón, O. y Piedra J. (2011). El patriarcado no es transparente: Competencias del profesorado para reconocer desigualdad. Cultura y Educación, 23(3), 385-397.

Freire, P. (1967). Pedagogía como práctica de la libertad. Siglo XXI.

Martínez, I. (2016). Construcción de una pedagogía feminista para una ciudadanía transformadora y contra-hegemónica. Foro de educación, 20, 129-151. https://10.14516/fde.2016.014.020.008

Martínez, I. (2018). Pedagogías feministas: estrategias para una educación emancipadora y decolonial. Revista Momento-Diálogos em Educaçao, 27(3), 350-365. https://doi.org/10.14295/momento.v27i3.8687

Martínez, I. y Bejarano, M.T. (2021). Educación en sexualidad e igualdad: conocimientos y desafíos en la formación docente. Revista Humanidades e innovaçao, 7(27), 134-148. https://revista.unitins.br/index.php/humanidadeseinovacao/article/ view/5161

Martínez, I., Bejarano, M.T., Marí, R. y Téllez, V. (2017). ¿Qué formación en sexualidad e igualdad tienen las y los estudiantes de educación en Castilla la Mancha? En J. Serrano, R. Moreno y S. Gutierrez (Coord..),Nuevos escenarios y retos socioeconómicos tras la crisis. Una perspectiva comparada de España y Europa (pp. 175-186). Dykinson.

Martínez, I. y Ramírez, G. (2017). Des-patriarcalizar y des-colonizar la educación: Experiencias para una formación feminista del profesorado. Revista Internacional de Educación para la Justicia Social (RIEJS), 6(2), 81-95. https://doi.org/10.15366/riejs2017.6.2.005.

Organización Mundial de la Salud. (2008). La salud sexual y su relación con la salud reproductiva: un enfoque operativo. OMS
Pastor, I. y Acosta, A. (2016). La institucionalización de las políticas de igualdad de género en la Universidad española. Avances y retos. Investigaciones Feministas, 7(2), 247-271.

Platero, L. (2014). Trans sexualidades. Acompañamiento, factores de salud y recursos educativos. Bellaterra.

Ramos, F., Martínez, I., Blanco, M. (2020). Sentido de la educación para la ciudadanía desde pedagogías feministas, críticas y decoloniales. Una propuesta para la formación del profesorado. Revista Izquierdas, 49, 2103-2116. http://izquierdas.cl/ ediciones/2020/numero-49

Sánchez, M., Penna, M. y de la Rosa, B. (2016). Somos como somos. Deconstruyendo y transformando la escuela. La Catarata.

Sánchez, M. (2020). Pedagogías queer. La Catarata.

Sánchez, M. (2018). Estudio integral de la violencia de género. Tirant.

Sánchez, R.; Buzón, O.; Piedra J. (2014). El patriarcado no es transparente: Competencias del profesorado para reconocer desigualdad. Cultura y Educación, 23(3), 385-397. https://doi. org/10.1174/113564011797330298

Segato, R. (2018). Contra-pedagogías de la crueldad. Prometeo.

Subirats, M. (2017). Coeducación. Apuesta por la libertad. Octaedro.

Teixeira, F. y Marques, F. (2016). Sexualidad y Género en la formación inicial de los docentes. En A. Mateos y A. Manzanares (Coord.), Mejores maestros, mejores educadores. Innovación y propuestas en educación (pp. 341-356). Aljibe.

UNESCO. (2014). Educación integral de la sexualidad. Conceptos, enfoques y competencias. Unesco

Veiga, L., Teixeira, F., Martinez, I. y Meliço-Silvestre, A. (2006). Sexuality and human reproduction: a study of scientific knowledge, behaviours and beliefs of Portuguese future elementary school teachers, Sex Education, 6(1), 17-29. https://doi. org/10.1080/14681810500508915 